\title{
A NEW AIRBORNE DOPPLER LIDAR FOR BOUNDARY LAYER RESEARCH \& LES-BASED SIMULATION
}

\author{
P. Gasch ${ }^{1 *}$, A. Wieser ${ }^{1}$, N. Kalthoff ${ }^{1}$, U. Corsmeier ${ }^{1}$, T. Feuerle ${ }^{2}$ and C. Kottmeier ${ }^{1}$ \\ ${ }^{1}$ Institute of Meteorology and Climate Research, Karlsruhe Institute of Technology, Karlsruhe, Germany \\ ${ }^{2}$ Insitute of Flight Guidance, Technical University Brunswick, Brunswick, Germany \\ *Email:philipp.gasch@kit.edu
}

\begin{abstract}
Airborne Doppler lidar measurements are a valuable tool in meteorological research. A new airborne Doppler lidar system has been developed jointly by the Karlsruhe Institute of Technology (KIT) and the Technical University of Brunswick. The system is designed for use aboard a mediumrange turboprop aircraft, thereby targeting mesoscale flow phenomena. In order to optimize system setup and retrieval strategy, as well as evaluate measurement quality under turbulent conditions, a first LES-based airborne Doppler lidar simulator has been developed. The results provide detailed insight into airborne Doppler lidar wind profiling characteristics.
\end{abstract}

\section{INTRODUCTION}

Airborne Doppler lidar measurements can provide added value compared to ground-based measurements due to their flexibility allowing for targeted measurements at high spatial resolution [1]. Due to their benefits, various airborne Doppler lidar systems have been developed in the past. Most of the existing systems are based on long-range aircraft flying high and fast, thereby they are destined for sensing the free troposphere due to the coarser spatial resolution $[2,3]$. Some deployments of airborne Doppler lidar have been reported based on medium-range aircraft flying low and slow for wind profiling purposes $[4,5]$.

In a joint project by the Karlsruhe Institute of Technology (KIT) and the Technical University of Brunswick a new airborne Doppler lidar system for boundary layer research has been designed. The new system has a flexible scanning geometry, allowing for both wind profiling and Nadir measurements of the wind field.

Wind profiling at highest resolution in the boundary layer suffers from errors due to a violation of the homogeneous conditions assumed for retrieval, e.g. due to turbulence in the scanned volume. In order to investigate the sensitivity of the error characteristics to system setup and retrieval strategy, a first LES-based airborne Doppler lidar simulator (ADLS) has been developed. The ADLS has the advantage that the atmospheric input data during the measurement process are known exactly. Thereby, representativeness problems due to differing measuring geometries, complicating airborne Doppler lidar validation for commonly used tools (e.g. dropsondes, towers, other profilers), can be avoided [6,7]. Further, a wide range of system setups and retrieval strategies can be evaluated.

\section{THE NEW AIRBORNE DOPPLER LIDAR SYSTEM}

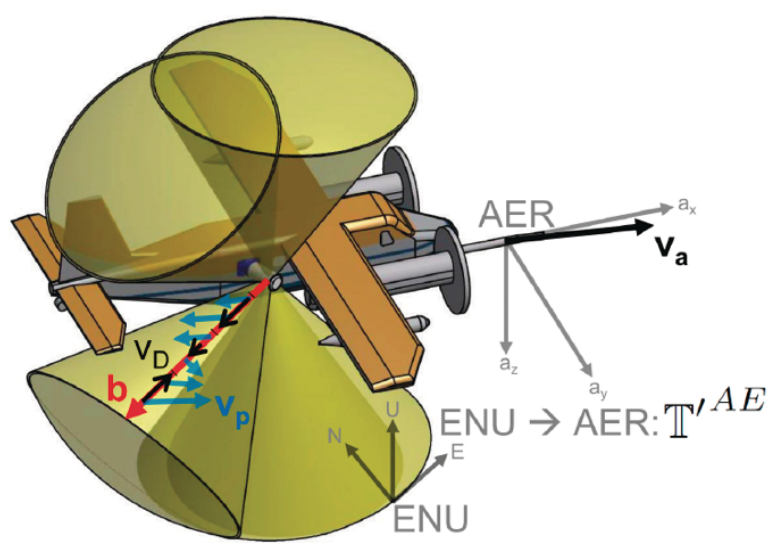

Figure 1: Schematic depiction of the airborne Doppler lidar system and idealized scan geometries. The coordinate systems used in the ADLS are indicated (ground based ENU, aircraft based AER). Further, the particle velocity $\mathbf{v}_{\mathbf{p}}$, beam pointing direction $\mathbf{b}$, measured radial Doppler velocity $v_{D}$ and aircraft velocity $\mathbf{v}_{\mathbf{a}}$ used in the simulations are also sketched.

A new airborne Doppler lidar (ADL) system is designed for use aboard the Dornier 128-6 research aircraft [8]. A Lockheed Martin WindTracer WTX Doppler lidar owned by KIT, measuring at $1.6 \mu \mathrm{m}$ wavelength, with a pulse duration of $300 \mathrm{~ns}$ and $5.4 \mathrm{~kW}$ peak power, is operated at a pulse repetition frequency of $750 \mathrm{~Hz}$ 
and $100 \mathrm{~ms}$ accumulation time. It is coupled with a new airborne scanner built by Arges GmbH. The 2-axis scanner can point both up and downward $\left( \pm 180^{\circ}\right.$ around the aircraft pitch axis), as well as $\pm 30^{\circ}$ sideway (swath) in all positions, at scan rates of $20^{\circ} \mathrm{s}^{-1}$. The system allows for flexible scanning geometries and automatic correction of aircraft movement. It yields high spatial resolution due to the slow flying aircraft $\left(65 \mathrm{~m} \mathrm{~s}^{-1}\right)$ and high measurement frequency.

In order to characterize the expected accuracy of wind profiling under inhomogeneous flow conditions, as well as to optimize system setup and retrieval strategies, an ADLS has been developed.

Table 1: System setup and retrieval parameters as used for this study.

\begin{tabular}{l|l}
\hline Parameter & Setting \\
\hline Aircraft altitude & $1700 \mathrm{~m}$ \\
Flight directions & $16,22.5^{\circ}$ apart \\
Scan elevation & $30^{\circ}-80^{\circ}$ \\
Scan rotation & $20 \mathrm{~s}$ \\
Data acquisition & $1 \mathrm{~Hz}$ \\
Components retrieved & $\mathrm{u}, \mathrm{v}, \mathrm{W}$ \\
Averaging distance & $1300 \mathrm{~m}$ \\
Vertical resolution & $62 \mathrm{~m}$ \\
Radial velocity noise $\sigma \mathrm{v}_{\mathrm{D}}$ & $0-1 \mathrm{~m} \mathrm{~s}^{-1}$ \\
Beam pointing error $\sigma \mathbf{b}$ & $0^{\circ}-1^{\circ}$ \\
\end{tabular}

\section{LES-BASED SIMULATION}

The ADLS allows for analysis of varying system setups as well as retrieval strategies by simulating the new ADL system numerically.

\subsection{Operating scheme}

The ADLS uses PArallelized LES Model (PALM) data as LES input $[9,10]$, which provides wind fields with $10 \mathrm{~m}$ grid-spacing at geostrophic background wind speeds of $\mathrm{u}_{\mathrm{G}}=0,5,10$ and 15 $\mathrm{m} \mathrm{s}^{-1}$. The surface kinematic heat flux is set to $0.03 \mathrm{~K} \mathrm{~m} \mathrm{~s}^{-1}\left(0.23 \mathrm{~K} \mathrm{~m} \mathrm{~s}^{-1}\right.$ for $\left.\mathrm{u}_{\mathrm{G}}=0 \mathrm{~m} \mathrm{~s}^{-1}\right)$. The setup results in an unstably stratified flow with the presence of organized convective structures. The turbulent boundary layer, wherein profiling is conducted, extends up to a height of $800 \mathrm{~m}$ (1400 $\mathrm{m})$. For wind profiling, the aircraft transects the LES 25 times in 16 directions in order to create a sufficient number of profiles. The scanner and lidar system are emulated geometrically, without simulation of the backscattering process or laser photonics. For the measurement, all geometric transformations applicable in real-world aircraft measurements are included in the ADLS. Two system simulations are performed: 1. Ideal measurement system, no error added on any component in order to focus on errors due to flow inhomogeneity. 2. Noisy measurement system, to emulate lidar and scanner error, random deviations can be added to the radial velocity ( $\sigma \mathrm{VD})$ and beam pointing direction $(\sigma \mathbf{b})$. The specific setup used for all components is shown in Tab. 1.

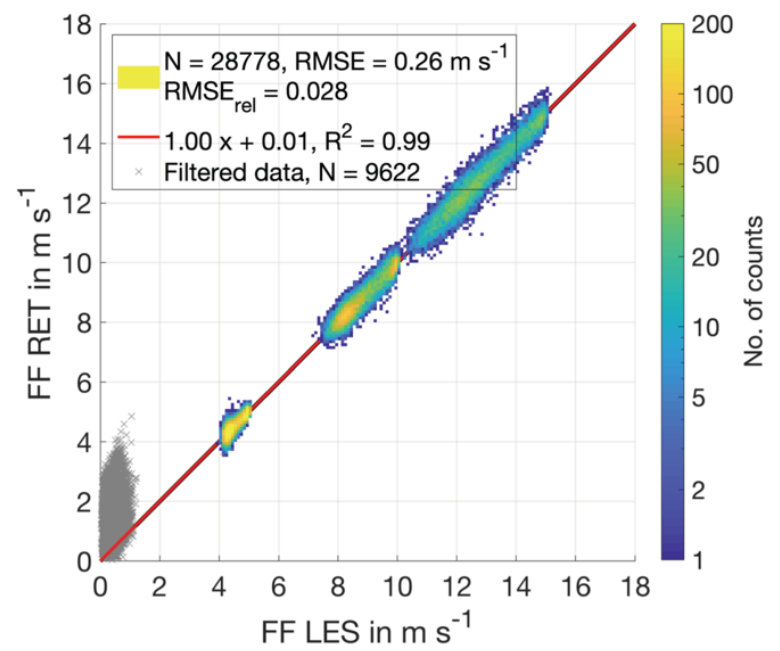

Figure 2: LES input wind speed (FF LES) and ADL retrieved wind speed (FF RET) for an ideal measurement system with specifications according to Tab. 1.

After the measurement, an airborne velocity azimuth display (AVAD) retrieval is performed on the measured data [11]. For error analysis, the originally supplied LES wind field data can then be evaluated against the retrieved wind data. For system setup and retrieval characterization, as well as optimization, a number of parameters adjustable in real-world measurements are varied to evaluate their effect on retrieval quality. Last, using the ADLS, it is possible to evaluate the performance of commonly used quality filtering criteria in Doppler lidar wind profiling. 


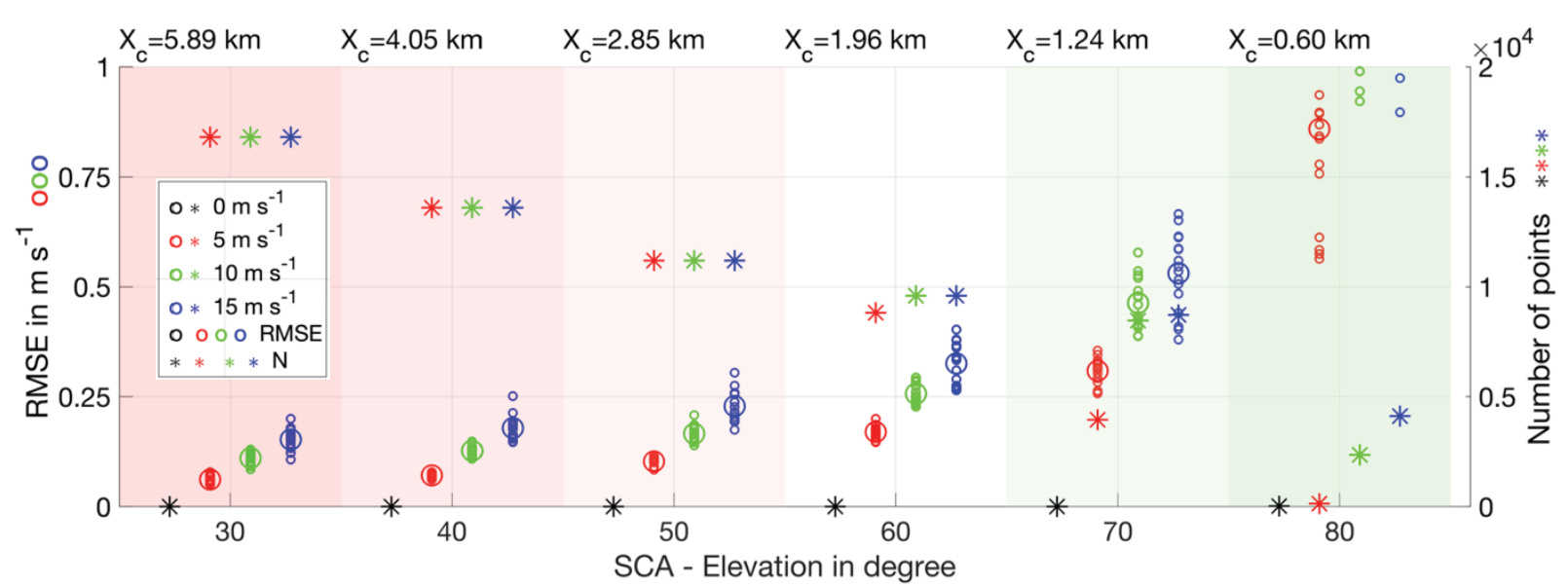

Figure 3: Root-mean-squared error (RMSE) and number of retrieved profiles for varying scan elevation angles and different background wind cases. All other parameters according to Tab. 1. For the $0 \mathrm{~m} \mathrm{~s}^{-1}$ background wind speed case no retrievals pass quality filtering.

\section{RESULTS}

\subsection{Error in wind profiling under turbulent conditions}

The comparison between LES input and ADL retrieved wind speeds for an ideal system is shown in Fig. 2. The overall retrieval quality is high for all wind speed cases, except the lowest $\left(\mathrm{RMSE}=0.26 \mathrm{~m} \mathrm{~s}^{-1}, \mathrm{R}^{2}=0.99\right)$. For the settings investigated, turbulence can cause considerable profiling error above $1 \mathrm{~m} \mathrm{~s}^{-1}$. A striking feature is that no useful retrieval is possible for the $0 \mathrm{~m} \mathrm{~s}^{-1}$ background wind speed case, where the retrieval is biased. However, all biased points are successfully eliminated by applying commonly used quality filtering criteria in the least-square fit analysis $\left(\mathrm{R}^{2}>0.9\right.$, condition number $\left.<9\right)$. The vertical distribution of profile retrieval error mirrors the underlying boundary layer turbulence (not shown). Profile error increases towards the middle of the boundary layer where amplitude and size of the inhomogeneities are largest. Towards the surface, a decrease in profiling error can be observed.

\subsection{Influence of system setup variation on retrieval error}

Using the ADLS, the parameters in Tab. 1 can be varied to investigate their influence on retrieval quality. A parameter that can be easily varied is the scan elevation angle. Steeper scan elevation angles (closer to nadir) allow for a smaller footprint of the measurement, but at the cost of decreasing the contribution of the horizontal wind to the measured radial velocity. Retrieval characteristics for varying scan elevation angles, using an idealized system (the new ADL allows for scans with elevation $<60^{\circ}$ only at forward and backward azimuth angles), can be evaluated from Fig. 3.

The results show that scanning at elevation angles steeper than $70^{\circ}$ is problematic for the conditions and setup investigated, as the retrieval error becomes large. When using more shallow elevation angles retrieval quality is improved, however, at the cost of having a much wider measurement footprint, denoted by the acrosstrack measurement diameter $\mathrm{x}_{\mathrm{c}}$. From this analysis, the usage of a $60^{\circ}$ scan elevation angle provides a reasonable tradeoff between profiling error and measurement footprint.

\subsection{Influence of system errors on retrieval quality}

Using the ADLS, it is possible to evaluate the influence of various error sources on wind profiling quality. The two system errors considered here are random radial velocity noise $\sigma V_{D}$ (e.g. due to detector noise, speckle effects and turbulence within the measurement volume) and beam pointing error $\sigma \mathbf{b}$ (due to scanner or aircraft positioning error). Both are varied using worst-case assumptions, the results are shown in Fig. 4.

Results show that turbulence is the main driver of profiling error for the higher wind speed cases. Even the worst-case error assumptions do not lead to retrieval error of comparable magnitude to that due to turbulence (Fig. 4). For the $5 \mathrm{~m} \mathrm{~s}^{-1}$ 
background wind speed case, system accuracy becomes important. Here an increase of error above $0.25 \mathrm{~m} \mathrm{~s}^{-1}$ or $0.25^{\circ}$ does not allow for retrieval of meaningful wind profiles anymore. Further, quality filtering seriously degrades the number of retrieved profiles for these cases. However, the error levels of $0.25 \mathrm{~m} \mathrm{~s}^{-1}$ and $0.25^{\circ}$ already present levels which are not expected to occur in a real system [4].
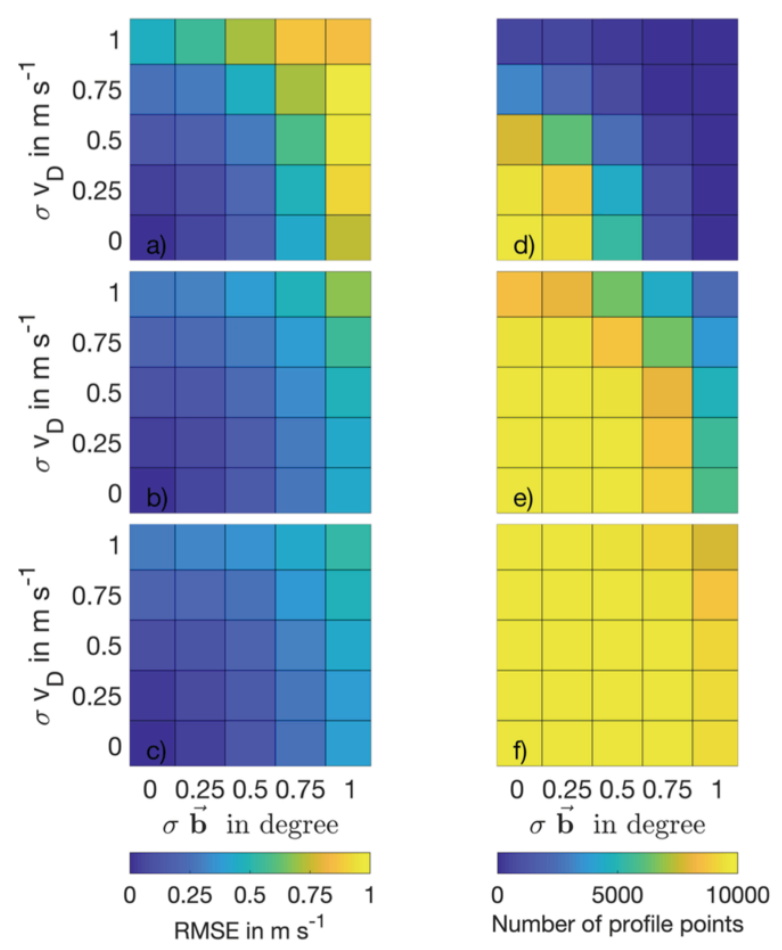

Figure 4: Retrieval error due to emulated noisy measurement system. a), d) $5 \mathrm{~m} \mathrm{~s}^{-1}$; b), e) $10 \mathrm{~m} \mathrm{~s}^{-1}$; c), f) $15 \mathrm{~m} \mathrm{~s}^{-1}$ background wind speed case. Retrieval error due to turbulence (determined using ideal system) has been subtracted from all entries.

\section{CONCLUSIONS}

A new airborne Doppler lidar system is designed for use aboard a medium-range twin-engine unpressurized turboprop aircraft. The new system is expected to provide insight into meso-scale flow phenomena. In preparation, for evaluation of wind profiling accuracy under inhomogeneous flow conditions, a first LES-based airborne Doppler lidar simulator has been developed. Results show that wind profiling is possible with high resolution in the boundary layer, except for situations where there is no background wind speed. Turbulence is the main driver of profiling error for higher wind speeds, at lower wind speeds system accuracy becomes important. Profiling error can be minimized through appropriate system setup and retrieval strategy. For optimization, the ADLS provides a valuable link between theory and measurements.

\section{ACKNOWLEDGEMENTS}

The first author acknowledges helpful remarks and comments on the LES-based simulator work by Prof. J. K. Lundquist.

\section{REFERENCES}

[1] Baker, W. et al.: Lidar-measured wind profiles: The missing link in the global observing system, Bull. Am. Meteorol. Soc., 95, 543-564, 2014.

[2] Chouza, F. et al.: Saharan dust long-range transport across the Atlantic studied by an airborne Doppler wind lidar and the MACC model, Atmos. Chem. Phys., 16, 11581-11600, 2016.

[3] Bucci, L. R., et al.: Validation of an Airborne Doppler Wind Lidar in Tropical Cyclones, Sensors, 18, 4288, 2018.

[4] De Wekker, S. F., et al.: Airborne Doppler lidar measurements of valley flows in complex coastal terrain, J. Appl. Meteorol. Climatol., 51, 1558-1574, 2012.

[5] Koch, G. J., et al.: Three-dimensional wind profiling of offshore wind energy areas with airborne Doppler lidar, J. Appl. Remote Sens., 8, 083662, 2014.

[6] Weissmann, M., et al.: Targeted observations with an airborne wind lidar, J. Atmos. Ocean. Technol., 22, 1706-1719, 2005.

[7] Wainwright, C. E., et al.: A time series sodar simulator based on large-eddy simulation, J. Atmos. Ocean. Technol., 31, 876-889, 2014.

[8] Corsmeier, U., et al.: Airborne turbulence measurements in the lower troposphere onboard the research aircraft Dornier 128-6, D-IBUF, Meteorol. Zeitschrift, 10, 315-329, 2001.

[9] Raasch, S. and Schröter, M.: PALM - A large-eddy simulation model performing on massively parallel computers, Meteorol. Zeitschrift, 10, 363-372, 2001.

[10] Stawiarski, C., et al.: Assessment of Surface-Layer Coherent Structure Detection in Dual-Doppler Lidar Data Based on Virtual Measurements, Boundary-Layer Meteorol., 156, 371-393, 2015.

[11] Leon, D. and Vali, G.: Retrieval of threedimensional particle velocity from airborne doppler radar data, J. Atmos. Ocean. Technol., 15, 860-870, 1998. 